This is the peer-reviewed version of the following article:

Trmčić, Milena, Hodgson, David R.W., 2011. Synthesis of thiophosphoramidates in water: Click chemistry for phosphates. Chemical Communications. 47, 6156-6158. https://doi.org/10.1039/C1CC11586C

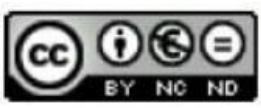

This work is licensed under a Creative Commons - Attribution-Noncommercial-No Derivative Works 3.0 Serbia 


\section{Durham Research Online}

\section{Deposited in DRO:}

06 November 2014

\section{Version of attached file:}

Accepted Version

Peer-review status of attached file:

Peer-reviewed

\section{Citation for published item:}

Trmčić, M. and Hodgson, D.R.W. (2011) 'Synthesis of thiophosphoramidates in water : click chemistry for phosphates.', Chemical communications., 47 (21). pp. 6156-6158.

\section{Further information on publisher's website:}

http://dx.doi.org/10.1039/c1cc11586c

Publisher's copyright statement:

Additional information:

Use policy

The full-text may be used and/or reproduced, and given to third parties in any format or medium, without prior permission or charge, for personal research or study, educational, or not-for-profit purposes provided that:

- a full bibliographic reference is made to the original source

- a link is made to the metadata record in DRO

- the full-text is not changed in any way

The full-text must not be sold in any format or medium without the formal permission of the copyright holders.

Please consult the full DRO policy for further details. 


\title{
Synthesis of Thiophosphoramidates in Water: Click Chemistry for Phosphates
}

\author{
Milena Trmčić and David R. W. Hodgson* \\ Received (in XXX, XXX) Xth XXXXXXXXX 200X, Accepted Xth XXXXXXXXX 200X \\ ${ }_{5}$ First published on the web Xth $X X X X X X X X X 200 X$ \\ DOI: $10.1039 / b 000000 x$
}

\begin{abstract}
An aqueous method for the preparation of $N, S$-dialkyl thiophosphoramidates is reported. Thiophosphorylation of alkylamines was performed using $\mathrm{SPCl}_{3}$ in aqueous reaction 10 media, and the resulting thiophosphoramidate-S-anions were $S$ alkylated with soft electrophiles. Ranges of amines and electrophiles were explored.
\end{abstract}

Phosphate esters and their structural analogues represent a major class of biomolecules that plays central roles in genetic 15 transmission, membrane formation, signalling and metabolism. The synthesis of phosphate esters and their analogues has been revolutionized by the phosphoramidite method. ${ }^{1}$ However, the preparation of phosphoramidites is often time-consuming, requiring global protection and 20 anhydrous conditions. Thereafter, the formation of phosphate esters requires anhydrous conditions and a range of reagents that are highly effective in automated oligonucleotide synthesizers, but cumbersome in general laboratory usage.

We sought to overcome these limitations by developing ${ }_{25}$ aqueous "click" methods that employ off-the-shelf reagents for the preparation of $N, S$-dialkyl thiophosphoramidates that represent simple mimics of phosphate diesters. Building on the use of phosphoryl chloride $\left(\mathrm{OPCl}_{3}\right)$ for the preparation of $N$-phosphorylated amines, ${ }^{2-4}$ we began to investigate the use 30 of thiophosphoryl chloride $\left(\mathrm{SPCl}_{3}\right)$ for the preparation of $\mathrm{N}$ thiophosphorylated amines, which could then be elaborated, through $S$-alkylation, to give $N, S$-dialkyl thiophosphoramidates (Scheme 1). A key aim was to ensure clean conversions, where the requirement for time-consuming 35 ion exchange or HPLC purifications that can often hamper the preparation of phosphate esters was significantly reduced or removed.

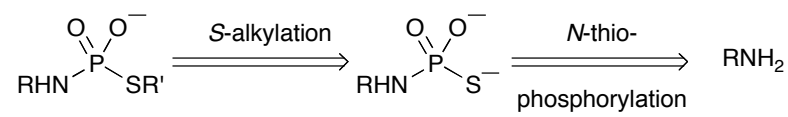

Scheme 1 Retrosynthetic strategy for the preparation of $N, S$-dialkyl thiophosphoramidates in aqueous solvent mixtures.

Our approach hinges on exploiting the greater intrinsic nucleophilicities of amines in comparison to water. Thus, through control of amine concentrations and other experimental parameters, we hoped to be able to induce 45 selective $N$-thiophosphorylation of amines versus hydrolysis of the thiophosphorylating agent. Similar approaches have been used for the preparation of carboxylic amides and sulfonamides, ${ }^{5}$ but, surprisingly, this approach has not been exploited significantly with (thio)phosphoric amide systems.
50 We first explored conditions for thiophosphorylation of the model amines ethanolamine and benzylamine with $\mathrm{SPCl}_{3}$ (Scheme 2 and Table 1).

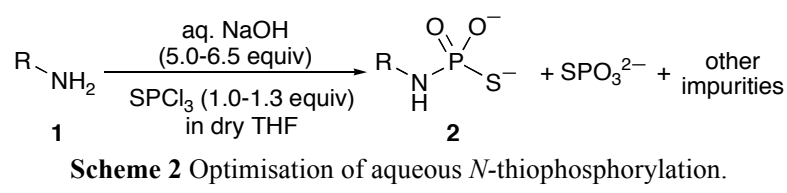

\begin{tabular}{|c|c|c|c|}
\hline entry & substrate 1 & eq. of $\mathrm{SPCl}_{3}$ & $2, \%$ \\
\hline 1 & & 1 & $98^{b}, 94^{c}$ \\
\hline 2 & & 1.1 & $96^{b}, 93^{c}$ \\
\hline 3 & & 1.2 & $92^{b}, 86^{c}$ \\
\hline 4 & & 1.3 & $91^{b}, 94^{c}$ \\
\hline 5 & $\mathrm{NH}_{2}$ & 1.0 & $97^{b},>99^{c}$ \\
\hline
\end{tabular}

55 Table 1 Screening thiophosphorylation conditions ${ }^{a}$

${ }^{a}$ See Supporting Information for details. ${ }^{b}$ Determined by ${ }^{31} \mathrm{P}$ NMR. ${ }^{c}$ Determined by ${ }^{1} \mathrm{H}$ NMR.

We used THF as a co-solvent given that $\mathrm{SPCl}_{3}$ appears to display very limited solubility in water. In all cases, 60 satisfactory conversions (mostly $>90 \%)$ to thiophosphoramidate 2 were obtained. Varying the numbers of equivalents of $\mathrm{SPCl}_{3}$, aiming to enhance conversions in the case of ethanolamine, afforded no improvements (entries 2-4). We also explored the number of equivalents of $\mathrm{NaOH}$ ${ }_{65}$ employed, but we found that 5 equiv represented the optimum number (data not shown). The only significant by-product from these $N$-thiophosphorylation procedures was inorganic thiophosphate, which likely arose because of the use of high concentrations of hydroxide ion causing competitive 70 hydrolysis of the thiophosphorylating agent. When using nonpolar amines, that could be readily extracted into organic solvents, the use of an excess of the amine substrate over $\mathrm{SPCl}_{3}$ helped to mitigate against this problem (data not shown). Successful $N$-thiophosphorylation using 1 equiv of $75 \mathrm{SPCl}_{3}$ with 1.0-1.2 equiv of amine substrate prompted us to explore $S$-alkylation of the $N$-thiophosphoramidate ions 2 with MeI in a one-pot procedure (Scheme 3). In all cases (Table 2, entries 1-4), conversions to $N$-alkyl $S$ methylthiophosphoramidate after simple extraction of excess ${ }_{80}$ MeI were high (>90\%). 


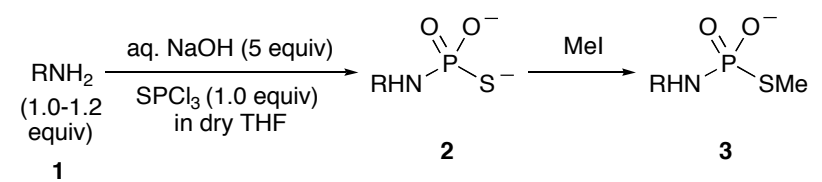

Scheme 3 Preliminary studies on $S$-alkylation of $N$-thiophosphoramidate ions using MeI.

Table $2 N$-Thiophosphorylation of amines and $S$-methylation of $N$ 5 thiophosphoramidates

\begin{tabular}{cc}
\hline entry & $\begin{array}{c}\text { conversion to } \mathrm{N} \text {-alkyl } S \text { - } \\
\text { methylthiophosphoramidate } \\
\mathbf{3}, \%\end{array}$ \\
\hline 1 & $98^{a}, 94^{b}$ \\
$97^{a}, 96^{b}$ & $97^{a}, 91^{b}$ \\
\hline
\end{tabular}

Expanding the range of $S$-alkylating agents, we found that much less electrophilic alkylating agents were also effective 10 (Scheme 4).

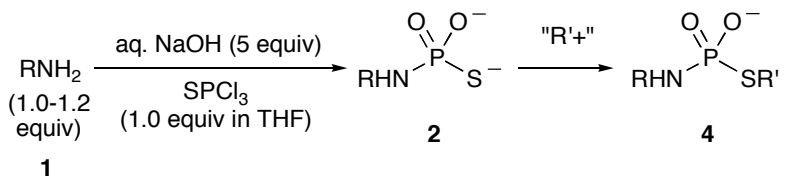

Scheme 4 Combined, one-pot $N$-thiophosphorylation $/ S$-alkylation using ranges of amines and alkylating agents.
Table $3 S$-alkylation of $N$-thiophosphoramidates

\begin{tabular}{cccc}
$\begin{array}{c}N \text {-alkyl- } \\
\text { thiophos- } \\
\text { phoramidate }\end{array}$ & entry alkylating agent & $\begin{array}{c}\text { reaction } \\
\text { time, } \mathrm{h}^{a}\end{array}$ & $\begin{array}{c}\text { conversion to } N, S \text { - } \\
\text { dialkyl } \\
\text { thiophosphomidate }\end{array}$ \\
\hline $999^{b},>99^{c}$ \\
$>99^{b},>99^{c}$ \\
$>99^{b},>99^{c}$ \\
$92^{b}, 92^{c}$ \\
$\left(90^{b}, 89^{c}\right)$
\end{tabular}

${ }_{15}{ }^{a}$ All alkylations were performed at room temperature except where stated. The amine was employed at 1.2 equiv except for entry 16 , where 1.0 equiv was used. ${ }^{b}$ Determined by ${ }^{31} \mathrm{P}$ NMR. ${ }^{c}$ Determined by ${ }^{1} \mathrm{H}$ NMR. ${ }^{d}$ Alkylation reaction heated at $50{ }^{\circ} \mathrm{C}$. ${ }^{e}$ Additional $\mathrm{NaOH}$ was added with alkylating agent. ${ }^{f}$ Alkylating agent added over several portions. ${ }^{g}$

20 Alkylation reaction heated at $80{ }^{\circ} \mathrm{C} .{ }^{h}$ Reaction mixture was neutralized with $\mathrm{HCl}$ prior to solvent removal. ${ }^{i}$ A mixture of isomers $(63: 26)$ was obtained from opening of the epoxide ring, where attack at the less hindered end was favored. ${ }^{j}$ Morpholine was used in $20 \%$ excess over $\mathrm{SPCl}_{3}$ during the $\mathrm{N}$-thiophosphorylation step. ${ }^{j}$ Alkylating agent displays

25 poor solubility in organic solvents, therefore, excess alkylating agent was removed by chromatography.

Pleasingly, across a range of amine substrates and alkylating agents, including halides, epoxides and conjugate acceptors, conversions were extremely good (mostly $>90 \%)$. The only

30 exception to this was the use of $5^{\prime}$-deoxy-5'-iodoguanosine, where a combination of steric, electronic and solubility factors make the 5'-iodide remarkably unreactive. Even in this unfavourable case, reasonable conversion (82\%) was observed.

35 Our method allows for the facile, aqueous preparation of $N, S$-dialkyl thiophosphoramidates. Conversion levels are extremely high, and impurities are, in most cases, limited to small amounts of unconverted starting materials, and $S$ alkylated thiophosphate ions. Unlike many conventional 40 organic-solvent-based methods, we have used a predominantly aqueous organic solvent mixture that supports the intrinsic solubility of the ionic phosphoramidate products. Furthermore, given this intrinsic solubility, there is no 
requirement to remove the aqueous solvent after reactions, thus diminishing the environmental impact of the methodology. In addition, the reactions procedures are easy to perform using readily available materials, conversion levels 5 are high, obviating the need for chromatography, and sideproducts (inorganic thiophosphate, $\mathrm{NaCl}$ and excess $\mathrm{NaOH}$ ) are innocuous or readily neutralised. In summary, our method fulfils Sharpless's criteria for "Click" processes within the limits of the range of experiments that we have performed. ${ }^{6}$

10 These factors should be taken in the context of phosphate ester mimics normally presenting significant synthetic challenges. ${ }^{7-9}$

We thank Durham University for a studentship (M.T.) and the Royal Society for funds for an automated chromatography 15 system.

\section{Notes and references}

Department of Chemistry, Science Laboratories, Durham University, South Road, Durham, DH1 3LE, United Kingdom.

E-mail:d.r.w.hodgson@durham.ac.uk

$20+$ Electronic Supplementary Information (ESI) available: general experimental procedures and ${ }^{1} \mathrm{H}$ and ${ }^{31} \mathrm{P}$ NMR spectra of reaction products. See DOI: 10.1039/b000000x/

1 G. M. Blackburn, M. J. Gait, D. Loakes and D. M. Williams, eds., Nucleic Acids in Chemistry and Biology, 3 edn., RSC Publishing, Cambridge, 2006.

2 R. Duncan and D. G. Drueckhammer, Tetrahedron Lett., 1993, 34, 1733.

3 D. Williamson, M. J. Cann and D. R. W. Hodgson, Chem. Commun., 2007, 5096.

304 D. Williamson and D. R. W. Hodgson, Org. Biomol. Chem., 2008, 6, 1056.

5 J. F. King, R. Rathore, J. Y. L. Lam, Z. R. Guo and D. F. Klassen, J. Am. Chem. Soc., 1992, 114, 3028.

6 H. C. Kolb, M. G. Finn and K. B. Sharpless, Angew. Chem. Int. Ed., 2001, 40, 2004.

7 M. Ora, K. Mattila, T. Lonnberg, M. Oivanen and H. Lonnberg, J. Am. Chem. Soc., 2002, 124, 14364.

8 J. W. Gaynor, J. Bentley and R. Cosstick, Nat. Protoc., 2007, 2, 3122 .

409 J. W. Gaynor, M. M. Piperakis, J. Fisher and R. Cosstick, Org. Biomol. Chem., 2010, 8, 1463. 